\title{
Unilateral Isolated Pleural Effusion in Ovarian Hyperstimulation Syndrome
}

\author{
Ali Sami GÜRBÜZ1', Emel Ebru ÖZÇIMIEN², Necati ÖZÇIMEN \\ Konya, Turkey
}

\begin{abstract}
Ovarian hyperstimulation syndrome (OHSS) is an iatrogenic complication with symptoms ranging from mild to severe. Pleural effusion occurs in severe forms of OHSS; however, isolated pleural effusion is rare. Here, we report a case of OHSS with isolated pleural effusion at presentation and discuss OHSS in light of the literature.
\end{abstract}

Keywords: Ovarian hyperstimulation syndrome, Ovulation induction, Pleural effusion

Gynecol Obstet Reprod Med 2016;22(2):108-109 DOI: 10.21613/GORM.2016.482

\section{Introduction}

Ovarian hyperstimulation syndrome (OHSS) is an iatrogenic complication that occurs in $33 \%$ of ovarian stimulation cycles, with clinical manifestations ranging from mild to severe (1). The pathogenesis of OHSS is unknown; however, increased vascular permeability leading to hemoconcentration and inadequate end-organ perfusion is considered to be the primary pathophysiology $(2,3)$.

Pleural effusion has been reported in $10 \%$ of severe OHSS cases and is usually associated with marked ascites $(4,5)$. Isolated pleural effusion is rare in OHSS cases (5).

Here, we report a case of OHSS with isolated pleural effusion and discuss the condition in light of the literature.

\section{Case Report}

A 30-year-old nulligravid woman with 5 years of unexplained infertility and no significant past medical history or physical findings was referred to our in vitro fertilization clinic. On day 2 of her menstrual cycle, 11 antral follicles were observed on each ovary; her follicle-stimulating hormone (FSH), luteinizing hormone, and estradiol (E2) values were $5.28 \mathrm{mIU} / \mathrm{mL}, 2.12 \mathrm{mIU} / \mathrm{mL}$, and $20 \mathrm{pg} / \mathrm{mL}$, respectively. Her male partner's spermiogram parameters were normal.

\footnotetext{
${ }^{1}$ Novafertil IVF Center, Konya

2 Başkent University Faculty of Medicine Obstetrics and Gynecology Department, Konya

${ }^{3}$ Medicana IVF Center, Konya

Address of Correspondence: Emel Ebru Özçimen

Başkent University Faculty of Medicine Obstetrics and Gynecology Department Konya, Turkey eparlakyigit@yahoo.com

Submitted for Publication: 16.02. 2015

Accepted for Publication:

24. 04.2015
}

Ovarian stimulation was initiated with 225 IU of recombinant FSH for 5 days. The dose was increased to 300 IU on day 5 because of low E2 levels and a poor ovarian response. On day 10 after induction, two follicles reached 20 and $18 \mathrm{~mm}$ in diameter. The patient's peak E2 level was $1733 \mathrm{pg} / \mathrm{mL}$. Oocyte pick-up (OPU) was performed $36 \mathrm{~h}$ after the administration of 10000 IU of urinary human chorionic gonadotropin (hCG). A total of 12 oocytes were retrieved, and one embryo transfer (ET) was performed on day 3 after OPU.

The patient's $\beta$-hCG level was $278 \mathrm{IU} / \mathrm{mL}$ on day 12 after ET. The patient complained of dyspnea 2 days after $\beta$-hCG positivity was established. She was afebrile and tachycardic, and her weight had increased by $2 \mathrm{~kg}$. Her oxygen saturation was $97 \%$ on room air. She had shortness of breath, a cough, and chest pain, tachypnea, and decreased breathing sounds. The results of echocardiography and electrocardiography were normal. She did not have nausea, vomiting, or abdominal distension.

An abdominal ultrasound revealed no evidence of intraperitoneal fluid; however, the patient's ovaries were enlarged bilaterally (right: 50x85 mm; left: 52x89 mm). Her liver and renal function tests and electrolytes were normal, and her hematocrit was $42 \%$.

The patient and her family refused a chest $\mathrm{x}$-ray because of her pregnancy. An ultrasound examination revealed right pleural effusion and minimal pleural effusion on the left side. Approximately $1500 \mathrm{cc}$ of yellow-colored fluid was aspirated from the right side by thoracentesis, and an additional $3000 \mathrm{cc}$ of fluid was drained via a pigtail catheter over 3 days. The fluid was exudative with $40 \mathrm{~g} / \mathrm{L}$ protein. A pleural fluid culture was negative. Low-molecular-weight heparin was administered to prevent deep vein thrombosis.

An abdominal ultrasound was performed daily and no fluid was observed. After 3 days, the patient's dyspnea and cough 
resolved, her $\beta$-hCG value was $1285 \mathrm{IU} / \mathrm{mL}$, and the pigtail catheter was removed without complications. The patient was discharged 2 days after the pigtail catheter was removed and followed closely as an outpatient undergoing serial ultrasound surveillance for pleural effusion and dyspnea. She recovered fully without sequelae. At present, the patient is at 9 weeks gestation in an uncomplicated singleton pregnancy.

\section{Discussion}

OHSS is a serious iatrogenic complication of controlled ovarian hyperstimulation. The condition is usually self-limiting, but it may be life-threatening (6). OHSS is classified as mild, moderate, or severe. Mild OHSS symptoms are relatively common in induced cycles and include abdominal distention, mild nausea, vomiting, and diarrhea (7). Pleural or pericardial effusion is a manifestation of severe OHSS (8). Severe OHSS has been reported in fewer than $2 \%$ of patients. Early OHSS is related to the ovarian response to stimulation and is an acute effect of exogenous hCG administration, which generally occurs within 9 days of oocyte retrieval. In contrast, late OHSS occurs after the initial 10-day period and is related more to the endogenous hCG produced by an implanted embryo than to the ovarian response (9). The primary goal of ovulation induction is to induce pregnancy; however, if pregnancy occurs, OHSS tends to be more severe with a longer duration.

Herein we report a case of isolated unilateral pleural effusion as a symptom of severe OHSS. Isolated pleural effusion associated with OHSS was first described in 1975 (4); however, few cases have been reported since (10-12).

The pathogenesis of isolated pleural effusion without ascites is unknown; however, negative intrapleural pressure may pull fluid from the abdomen into the thoracic cavity through the holes in the diaphragm (13).

Previous studies have shown that pleural effusions may be exudative, as in our case, or transudative (8). Because our patient was pregnant, a chest $\mathrm{x}$-ray could not be performed; however, we used ultrasonography to detect pleural effusion. Ultrasound techniques have been shown to be useful for the diagnosis of pleural effusion (14).

Our patient did not have abdominal distension or ascites; thus, the pleural effusion may have been overlooked with an abdominal examination alone. The view that dyspnea is only a direct result of increased intra-abdominal pressure in patients with OHSS can mislead physicians.

Dyspnea, pleural effusion, ascites, and hemoconcentration are observed in severe cases of OHSS. Our patient had no significant markers of severe OHSS aside from massive pleural effusion. Although massive pleural effusion may be caused by several systemic diseases, we suspected OHSS because our patient was pregnant, and she improved following thoracente- sis and supportive therapy. Thus, a thorough examination of patients with OHSS coupled with effective supportive therapy may lead to a favorable prognosis. In OHSS cases without abdominal ascites, pleural effusion should be considered in the presence of dyspnea.

\section{References}

1. Royal College of Obstetricians and Gynaecologists, 'The management of ovarian hyperstimulation syndrome,' Green Top Guideline 5, Royal College of Obstetricians and Gynaecologists 2006.

2. Asch RH, Li HP, Balmaceda JP, Weckstein LN, Stone SC. Severe ovarian hyperstimulation syndrome in assisted reproductive technology: definition of high risk groups. Hum Reprod 1991;6(10):1395-9.

3. MacDougall MJ, Tan SL, Jacobs HS. In vitro fertilization and the ovarian hyperstimulation syndrome. Human Reprod 1992;7(5):597-600.

4. Rabinerson D, Shalev J, Royburt Z, Ben-Rafael, Dekel A. Severe unilateral hydrothorax as the only manifestation of the ovarian hyperstimulation syndrome. Gynecol Obstet Inves 2000;49(2):140-2.

5. Golan A, Ron-el R, Herman A, Soffer Y, Weinraub Z, Caspi E. Ovarian hyperstimulation syndrome: an updata review. Obstet Gynecol Survey 1989;44(6):430-40.

6. Yildizhan R, Adali E, Kolusari A, Kurdoğlu M, Ozgokce C, Adali F. Ovarian hyperstimulation syndrome with pleural effusion: a case report. Cases J 2008;18(1):323.

7. Whelan JG $3^{\text {rd }}$, Vlahos NF. The ovarian hyperstimulation syndrome. Fertil Steril 2000;73(5):883-96.

8. Junqueira JJ, Bammann RH, Terra RM, Pugliesi de Castro AC, Ishy A, Fernandez A. Pleural effusion following hyperstimulation. J Bras Pneumol 2012;38(3):400-3.

9. McNeary M, Stark P. Radiographic findings in ovarian hyperstimulation syndrome. J Thorac Imaging 2002;17(3): 230-2.

10. Hsieh MJ, Tsao TC, Cheng PJ. Ovarian hyperstimulation syndrome with minimal ascites and massive pleural effusion: report of a case. J Formos Med Assoc 1994;93(10): 882-4.

11. Bassil S, Da Costa S, Toussaint-Demylle, Lambert M, Gordts S, Donnez JA. Unilateral hydrothorax as the only manifestation of ovarian hyperstimulation syndrome: a case report. Fertil Steril 1996;66(6):1023-5.

12. Anderson K, Haxton M, Banham S, Black WP. Unilateral pleural effusion as the presenting feature of ovarian hyperstimulation syndrome. Scott Med J 1988;33(5):338-9.

13. Roden S, Juvin K, Homasson JP, Israël-Biet D. An uncommon etiology of isolated pleural effusion. The ovarian hyperstimulation syndrome. Chest 2000;118(1):256-8.

14. Lichtenstein DA. Lung ultrasound in the critically ill. Ann Intensive Care 2014; 4(1):1-152. 